OPEN ACCESS

Edited by:

Professor Zodwa Dlamini

University of Pretoria, South Africa

Reviewed by:

Dan Shao,

South China University of Technology,

China

Madhappan Santha Moorthy, Division of Metallic Biomaterials, Institute of Materials Research, Helmholtz Centre for Materials and Coastal Research, Germany

*Correspondence:

Yahya E. Choonara

yahya.choonara@wits.ac.za

Specialty section:

This article was submitted to

Nanobiotechnology,

a section of the journal

Frontiers in Molecular Biosciences

Received: 08 March 2021

Accepted: 19 April 2021

Published: 20 May 2021

Citation:

Freidus LG, Kumar $P$

Marimuthu T, Pradeep P and Choonara YE (2021) Theranostic Mesoporous Silica Nanoparticles Loaded With

a Curcumin-Naphthoquinone Conjugate for Potential Cancer Intervention.

Front. Mol. Biosci. 8:670792 doi: 10.3389/fmolb.2021.670792

\section{Theranostic Mesoporous Silica Nanoparticles Loaded With a Curcumin-Naphthoquinone Conjugate for Potential Cancer Intervention}

\author{
Lara G. Freidus, Pradeep Kumar, Thashree Marimuthu, Priyamvada Pradeep and \\ Yahya E. Choonara*
}

\begin{abstract}
Wits Advanced Drug Delivery Platform Research Unit, Department of Pharmacy and Pharmacology, School of Therapeutic Sciences, Faculty of Health Sciences, University of the Witwatersrand, Johannesburg, South Africa
\end{abstract}

A novel theranostic molecule, derived from curcumin (Cur) and naphthoquinone (NQ), allowing for cancer targeting, detection and treatment was previously described and termed CurNQ. To allow for enhanced theranostic capabilities, advanced drug delivery techniques are required. To this end, mesoporous silica nanoparticles (MSN) were synthesized and CurNQ was loaded into its pores to form the novel nanosystem MSN_CurNQ. The formation of the nanosystem aimed to augment the drug delivery of CurNQ through the EPR effect and sustained release. Moreover, the loading of CurNQ into its pores, formed a fluorescent nanoparticle that can be tracked, detected and visualized. Herein, the synthesis of a novel nanosystem is described and its theranostic potential are explored in vitro. MSN with an average size of $108 \mathrm{~d} . \mathrm{nm}$, a zeta potential of $-42 \mathrm{mV}$ and a PDI of 0.150 were synthesized and were impregnated with CurNQ to form the novel nanosystem MSN_CurNQ. MSN_CurNQ was demonstrated to have $\mathrm{pH}$ responsivity whereby after $96 \mathrm{~h}$, at $\mathrm{pH} 7.4,31.5 \%$ of CurNQ was released from the MSN compared to $57 \%$ release at $\mathrm{pH} 6.8$, corresponding to an increase of $25.5 \%$ in release with a $0.6 \mathrm{pH}$ drop. The innate fluorescence was then characterized through confocal and fluorescence microscopy. Microscopy images illustrated the distinct, high intensity innate fluorescence with a high background to target ratio, thus confirming detection capabilities and potentially extending MSN_CurNQ's application to molecular imaging purposes. Moreover, the chemotherapeutic potential of MSN_CurNQ was demonstrated as cell viability was reduced to below 50\% in OVCAR-5, CACO-2, CHLA, and MCF-7 cell lines. Furthermore, MSN_CurNQ displayed tumor specific toxicity whereby the cell viability was reduced to a far greater extent in the cancer cell lines compared to a healthy fibroblast cell line $(p=0.000)$. Indeed, the novel MSN_CurNQ nanosystem has potential for applications in cancer targeting, detection and treatment

Keywords: curcumin, naphthoquinone, cancer, mesoporous silica nanoparticles, theranostics, fluorescence 


\section{INTRODUCTION}

Cancer is set to become the leading cause of death globally and is the biggest impediment to increased life expectancy in the 21 st century as the global incidences and mortality rates are forever increasing (Bray et al., 2018). Even with the multitude of research and resources dedicated to cancer, the cancer burden has not eased (Maruthappu et al., 2017). Clearly, new and improved strategies for cancer diagnostics and targeted treatment is an immediate necessity.

The search for new chemical entities for cancer treatment is ongoing. New cancer therapies should circumvent the issues that plague current chemotherapeutics, some of which include, high toxicity, non-specificity, cancer resistance and late diagnosis (Tran et al., 2017; Haider et al., 2020). One of the current trends in cancer research is the development of advanced platforms using nanotechnology that allows for simultaneous diagnostics and treatment, this field of research is referred to as theranostics (Saroj and Rajput, 2018). Concurrent diagnostics and treatment would allow for earlier detection and treatment of malignancy, resulting in improved prognosis and lower mortality rates (Wicki et al., 2015).

Theranostic intervention is often achieved using nanotechnology. Nanomedicine has become of great interest in the field of cancer research on account of enhancements to drug delivery mechanisms (Shi et al., 2017). A significant barrier to cancer treatment is the non-specificity of treatment which induces severe side effects and reduces treatment efficacy ( $\mathrm{Hu}$ et al., 2016; Haider et al., 2020). The targeting of treatment to cancer cells would reduce systemic toxicity and increase treatment effectiveness and thus is of immense interest (Wicki et al., 2015). The tunable and controllable drug delivery that can be achieved using nanomedicine has propelled this field forward into the spotlight of cancer intervention (Youn and Bae, 2018).

Leaky tumor vasculature is hallmark feature of cancer. This induces nanoparticles to leak out of tumor blood vessels (Shi et al., 2017). This phenomenon does not occur in healthy vessels owing to well-organized branching and regularly spaced capillary beds. This highly structured vessel is not apparent in tumor vasculature on account of rapid tumor growth, allowing for the leaking of nanoparticles into the tumor interstitium. Concurrently, poor tumor lymphatic drainage enables nanoparticle accumulation and enhanced retention (Baeza et al., 2016; Tran et al., 2017). The automatic accumulation of nanoparticles in tumor tissue has been well documented and is referred to as the Enhanced Permeation and Retention (EPR) effect (Shi et al., 2017). This effect has been the rationale behind the field of nanomedicine for cancer intervention. EPR allows for the passive targeting of nanoparticles to tumor tissue (Ivey et al., 2016). The addition of an active targeting strategy in conjunction with EPR can allow for enhanced tumor targeting capabilities.

Mesoporous silica nanoparticles (MSN) are a class of nanoparticles that have been widely studied for drug delivery applications. Silica is one of the most abundant natural resources and has been deemed safe and is widely used in food additives, pharmaceutical products and cosmetics (Bernardos and Kouřimská, 2013). With the explosion into nanomedicine research, silica has been the focus of an abundance of research, mainly in terms of drug delivery (Moreira et al., 2016; Watermann and Brieger, 2017). MSN have numerous features that make them ideal for advanced drug delivery, some of which include their solid framework, high surface to volume ratio, are mesoporous for drug loading, their chemically inert nature, along with their biocompatibility, biodegradability and that they show no toxicity in vitro (Argyo et al., 2013; Mai and Meng, 2013; Ma'mani et al., 2014; Liu et al., 2016; Alyassin et al., 2020). Moreover, their hydrophilic silanol surface is ideal for the delivery of low solubility drugs and allows for easy surface functionalization (Mai and Meng, 2013). The large pores evenly distributed over the surface of MSN are ideal for drug loading, especially for the loading of hydrophobic drugs. The loading of hydrophobic drugs into MSN has become a standard approach in the delivery of hydrophobic drug molecules. The poor solubility of these drugs is overcome as EPR delivers these loaded drugs directly into the tumor (Ma'mani et al., 2014). These properties of MSN make them ideal for advanced drug delivery applications.

Our previous research demonstrated the development of a novel theranostic molecule for cancer intervention. The novel molecule termed CurNQ, was synthesized through a Williamson ether synthesis reaction between curcumin (Cur) and lawsone (Freidus et al., 2020). Cur and lawsone were selected owing to the plethora of research that has been conducted on their promising anticancer properties, as well as their highly aromatic structures, allowing for fluorescent properties. As per our reported research (Freidus et al., 2020), CurNQ exhibited theranostic properties with potential applications in simultaneous cancer targeting, detection and treatment. CurNQ was demonstrated to have $\mathrm{pH}$ specific solubility, whereby at $\mathrm{pH} 7.4$ it remained mostly insoluble, but experienced a large increase in solubility at the $\mathrm{pH}$ associated with the tumor microenvironment, $\mathrm{pH}$ 6.8. Thus, allowing for cancer targeting through the exploitation of the change in $\mathrm{pH}$ associated with malignant transformation (Warburg, 1925). Once soluble, CurNQ displayed intense fluorescence, further allowing for cancer detection. Additionally, CurNQ induced cytotoxicity to two ovarian cancer cell lines, thereby extending its application to cancer therapy. To allow for the use of CurNQ in clinical applications, CurNQ was required to be delivered to the tumor microenvironment and for its fluorescence to be detected. Consequently, the loading of CurNQ into a nanoparticle for enhanced drug delivery is expected to further the theranostic capabilities of CurNQ allowing for enhanced targeting, sustained drug delivery and detection of the fluorescence nanoparticle.

The aim of this research was to further the theranostic capabilities of the previously described novel molecule CurNQ through the synthesis of mesoporous silica nanoparticles and the loading of CurNQ into its pores (MSN_CurNQ). This aimed to form a detectable fluorescent nanoparticle that has additional targeting and therapeutic properties. 


\section{MATERIALS AND METHODS}

\section{Materials}

CTAB-Hexadecyltrimethylammonium bromide (MW-364.45), Pluronic F-127 (MW-12600), TEOS-Tetraethyl orthosilicate (MW-208.33), Phalloidin-Atto 488 (MW- 1472) and DAPI4',6-Diamidine- $2^{\prime}$-phenylindole dihydrochloride (MW- 350.25) were purchased from Merck/Sigma Aldrich (Darmstadt, Germany) and were used as received. High purity solvents (> 98\%) were purchased and used as received from ACE chemicals (Johannesburg, South Africa). Cell lines were all acquired from Cell Biolabs Inc. (San Diego, United States) and from Fox Chase Cancer Center (Philadelphia, United States). Cell culture consumables, MTT-3- (4,5-dimethylthiazol-2-yl)2,5-diphenyl tetrazolium bromide, and other cell culture reagents were sourced from Thermo Fischer Scientific (Waltham, United States).

\section{Mesoporous Silica Nanoparticle Synthesis}

The synthesis of mesoporous silica nanoparticles (MSN) was performed using a modified method establish by Yang et al. and the synthesis scheme is displayed in Figure 1 (Yang et al., 2006). Equal concentrations of CTAB and surfactant (Pluronic F127) were dissolved completely in deionized water. $2 \mathrm{M}$ sodium hydroxide was added into the solution and then the temperature of the solution was increased and maintained at $80^{\circ} \mathrm{C} .6 \mathrm{~mL}$ of TEOS was added dropwise at a steady drop rate into the solution and then the solution was stirred rapidly whilst maintaining the temperature at $80^{\circ} \mathrm{C}$ for $2 \mathrm{~h}$. Subsequently, the solution was immediately filtered and left to dry for $24 \mathrm{~h}$ at ambient temperature. The remaining white powder was then collected and calcined at $550^{\circ} \mathrm{C}$ for $5 \mathrm{~h}$ in a furnace to remove the unwanted surfactants.

\section{Determination of MSN Size and Zeta Potential}

Size and zeta potential analyses were performed on the Zetasizer Nano ZS (Malvern, United Kingdom.). Dilute $10 \mathrm{~mL}$ suspensions

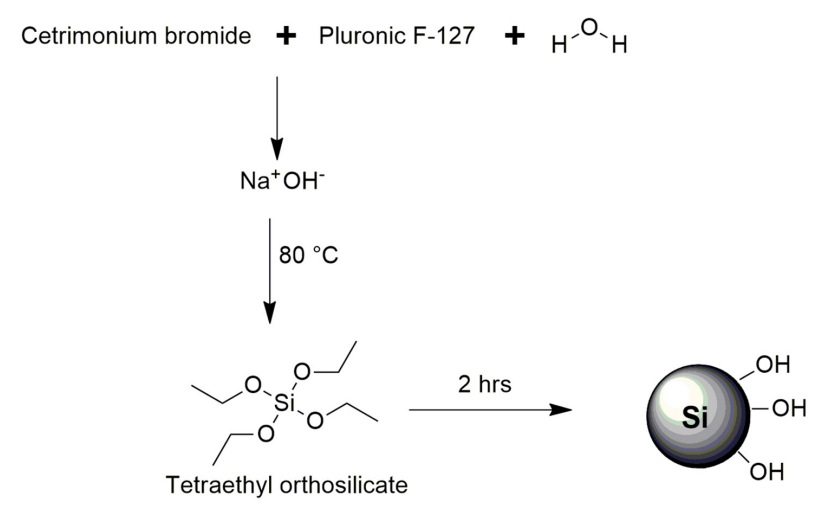

FIGURE 1 | Reaction scheme for the synthesis of MSN. of nanoparticles were sonicated for $30 \mathrm{~min}$ at room temperature prior to analysis to reduce particle aggregation. $2 \mathrm{~mL}$ of the dispersed samples were filtered into disposable cuvettes and the size, polydispersity index (PDI) and zeta potential values were recorded at $25^{\circ} \mathrm{C}$ on the ZetaSizer Nano ZS.

\section{X-Ray Diffraction-Crystallinity Characterization}

$\mathrm{X}$-ray diffraction (PXRD) utilizing a variable and fixed slit system was used to determine the crystallinity and atomic spacing and unit cell dimensions of the MSN. This characterization technique was performed on the Rigaku MiniFlex 600 Benchtop X-ray Diffractometer (Rigaku Corporation, Tokyo, Japan). A powdered sample was packed onto the sample holder and was analyzed at a scanning rate of $15^{\circ}$ per minute at a diffraction angle range of $3-90^{\circ}$ with a degree step of 0.02 , a voltage of $40 \mathrm{kV}$ and a current of $15 \mathrm{~mA}$.

\section{Porosity and Surface Area Determination}

Surface area and porosity analysis was performed on MSN using Porositometric Analyzer (Micromeritics ASAP 2020, Norcross, GA, United States). Surface moisture and contaminants were removed through degassing prior to analysis through the insertion of a glass filter rod. Degassing occurred over a period of $9 \mathrm{~h}$ and after the degassing process, the MSN were transferred to the analysis port where surface area, pore volume, and pore size data were obtained in accordance with standard BJH and BET gas adsorption method computations (Bawa et al., 2011).

\section{MSN Morphological Characterization}

The FEI Nova Nanolab 600 SEM was utilized for scanning electron microscopy (SEM) which was used to determine nanoparticle morphology and offer particle size confirmation through dynamic light scattering. Powdered sample of the nanoparticles was placed onto an aluminum specimen stub covered with a double-sided carbon adhesive disk and placed under a vacuum, flushed with argon, evacuated and sputter coated with gold for $4 \mathrm{~min}$ at $20 \mathrm{kV}$. SEM images were obtained on a FEI Nova Nanolab 600 FEG-SEM/FIB.

\section{Loading of CurNQ Into MSN to Create Novel Nanosystem}

Mesoporous silica nanoparticles were impregnated with CurNQ and were designated MSN_CurNQ. To achieve this, the methodology previously utilized by Jambhrunkar et al. to load Cur into MSN was followed owing to similarity (Ma'mani et al., 2014). Here $40 \mathrm{mg}$ of CurNQ was dissolved in methanol and mixed with $160 \mathrm{~g}$ of MSN particles (1:4 ratio) rapidly for $24 \mathrm{~h}$ at room temperature protected from light. The solvent was then slowly removed under reduced pressure using a rotary evaporator, further encouraging CurNQ entrapment. The particles were washed thrice with ethanol and twice with deionizer water to remove unentrapped CurNQ and loaded particles were collected via centrifugation at $5,000 \mathrm{rpm}$ for $10 \mathrm{~min}$. The particles were then dried in an oven overnight at $37^{\circ} \mathrm{C}$. The above procedure was repeated 
with Cur to produce MSN_Cur, which was to be used for comparative analyses.

\section{Determination of Encapsulation Efficiency of CurNQ Within MSN}

A mixture containing $40 \mathrm{mg}$ of CurNQ and $160 \mathrm{mg}$ of MSN was added to methanol and was stirred rapidly, allowing for loading to occur (Ma'mani et al., 2014). The UV absorbance of the solution was measured at $429 \mathrm{~nm}$ for CurNQ and $425 \mathrm{~nm}$ for Cur at varying time points over $48 \mathrm{~h}$. A decrease in absorbance over $48 \mathrm{~h}$ was recorded and the concentration of drug uptake was calculated using a standard curve of concentration versus UV absorbance at 429 and $425 \mathrm{~nm}$, respectively $\left(R^{2}=0.99\right)$.

Entrapment efficiency values of 54.54 and $32.5 \%$ were computed for CurNQ and Cur, respectively. A decrease in UV absorbance indicated successful entrapment owing to CurNQ being impregnated into MSN thereby being removed from the solution.

\section{Determination of CurNQ Release Profile From MSN at Varied pH}

CurNQ release from the MSN nanoparticles was measured at $\mathrm{pH} 7.4$ and 6.8 at $37^{\circ} \mathrm{C}$ for both MSN_CurNQ and MSN_Cur. One $\mathrm{mg} / \mathrm{mL}$ of CurNQ-loaded nanoparticles were immersed in a $\mathrm{PBS} / \mathrm{CTAB}$ solution and the $\mathrm{pH}$ of each sample was adjusted to mimic both physiological conditions ( $\mathrm{pH}$ 7.4) and at the tumor microenvironment ( $\mathrm{pH}$ 6.8). Samples were incubated and stirred on an orbital shaker incubator at a rate of $50 \mathrm{rpm}$ at $37^{\circ} \mathrm{C}$. At 12 $\mathrm{h}$ intervals, $1 \mathrm{~mL}$ of buffer was removed, and UV absorbance was measured through UV/Vis spectroscopy at $429 \mathrm{~nm}$. Total CurNQ release was then calculated using a standard curve $\left(R^{2}=0.99\right)$ for both CurNQ and Cur, respectively. The experiment was performed in triplicate to ensure statistical reliability $(N=3)$. The experiment was concluded after 96-h as no more CurNQ was released after this time.

\section{In vitro Determination of Chemotherapeutic Potential of MSN_CurNQ}

Multiple cell lines were used to determine the cytotoxic effects of MSN_CurNQ and MSN_Cur on a variety of cancer types. The following cell lines were cultured namely: OVCAR-5, 3T3, MCF7, HeLa, CACO-2, HT-29 and CHLA. The cell lines were cultured using standard tissue culture techniques. Cells were either grown in RMPI 1,640 or DMEM, depending on the guidelines specified by the cell line supplier. All media was supplemented with $10 \%$ foetal bovine serum (FBS) and 1\% Streptomycin and penicillin (Freidus et al., 2020). Cells were incubated at $37^{\circ} \mathrm{C}$ at $5 \% \mathrm{CO}_{2}$ atmospheric pressure. Once cells reached $80 \%$ confluency, cells were detached and sub-cultured using trypsin $(0.05 \% \mathrm{w} / \mathrm{v})$ and $\operatorname{EDTA}(0.01 \% \mathrm{w} / \mathrm{v})$.

\section{Cytotoxicity Studies Through MTT Assays}

Seven cell lines were cultured and the cytotoxicity of MSN_CurNQ and MSN_Cur was evaluated on all seven cell lines, thus allowing for the cytotoxic effect of MSN_CurNQ to be evaluated on several differing cancer types. Cells were cultured as per above conditions. Cell confluency was maintained at $70-80 \%$ through standard sub-culturing techniques utilizing trypsin/EDTA harvesting. Thereafter, $1.5 \times 10^{4}$ cells were counted and distributed equally in a 96 well dish and were incubated at $37^{\circ} \mathrm{C}$ in $5 \% \mathrm{CO}_{2}$ for $24 \mathrm{~h}$, allowing for cell attachment. Subsequently, cells were treated with varying concentrations of MSN_CurNQ and MSN-Cur. Cells were incubated in treatments for 24,48 , and 72 -h time period. After which, 3- (4,5- dimethylthiazol-2-yl)-2,5-diphenyl tetrazolium bromide (MTT) reagent was pipetted into each well and was incubated $4 \mathrm{~h}$. DMSO was then used to solubilize the formazan crystals. All samples were performed in quadruplicate for statistical reliability. Absorbance readings were then recorded on a Perkin Elmer Victor X3 2030 multilabel reader at $570 \mathrm{~nm}$ and $690 \mathrm{~nm}$.

\section{Determination of Fluorescent Properties of MSN_CurNQ Using Confocal Microscopy}

The OVCAR-5 cell line was cultured on coverslips until reaching $70 \%$ confluency. Cells were then treated with MSN_CurNQ for $24 \mathrm{~h}$, thereby allowing for cellular uptake to occur. After this, Phalloidin-Atto 488 was added, and staining occurred over $30 \mathrm{~min}$. Cells were then washed and fixed through incubation in 5\% formaldehyde in PBS for $20 \mathrm{~min}$. After fixation, DAPI stain was added at a concentration of $1 \mu \mathrm{g} / \mathrm{mL}$ and samples were left to incubate for $15 \mathrm{~min}$ in the dark. Hereafter, samples were washed thoroughly with PBS to remove unstained DAPI. Coverslips were stored in the dark in PBS. Coverslips were viewed on the Zeiss Laser Scanning Confocal Microscope (LSM) 780 (Oberkochen, Germany). Super resolution microscopy was performed using Airyscan technology as an added on feature to the LSM confocal microscope.

\section{Cytotoxicity Studies Using High Content Imaging System}

Exploiting the use of advanced technological systems such as machine learning, and AI has rapidly advanced the field of cell biology and enables quantitative and qualitative data to be extracted through microscopy images (Wedege et al., 2016). Indepth and detailed data was obtained through rapid microscopy image acquisition and the application of complexed algorithms which were applied using a high content imaging system and provided detailed quantitative and qualitative data on cellular events after treatment with the novel nanosystem. Cells were detached from flasks using EDTA/trypsin and were transferred and distributed equally to a 96-well dish and were then incubated under standard conditions overnight. Cells were then treated with MSN_CurNQ and MSN_Cur at varying concentrations, and cells were left in treatment for $24 \mathrm{~h}$ after which, cells were washed with PBS, fixed in 5\% formaldehyde for $20 \mathrm{~min}$ and then incubated in a $1 \mu \mathrm{g} / \mathrm{mL}$ solution of DAPI for $15 \mathrm{~min}$. Subsequently, cells were thoroughly washed in PBS thus removing residual unbound dye. The 96-well dish was then 
immediately inserted into the Logos Biosystem CELENA ${ }^{\circledR} \mathrm{X}$ High Content Imaging System which obtained rapid fluorescent microscopy images of each well, and quantitative and qualitative data was obtained from these images through the use of their proprietary software (Geonggi-do, South Korea).

\section{RESULTS AND DISCUSSION}

\section{Synthesis and Characterization of Mesoporous Silica Nanoparticles}

Mesoporous silica nanoparticles (MSN) were synthesized and were aimed to act as a delivery platform for CurNQ (Mai and Meng, 2013). Small particles with an average size around 100 d.nm with a substantial negative zeta potential were desired, allowing for the EPR effect as well as for the colloidal stability of the particles. A modified method from Yang et al. (2006) allowed for the synthesis of MSN with the desired characteristics.

As shown in Figure 2, the average size of the synthesized MSN was determined to be $108 \mathrm{~d}$.nm with a polydispersity index (PDI) of 0.150 . Moreover, the zeta potential of the nanoparticles was determined to be $-42 \mathrm{mV}$, confirming the colloidal stability of the nanoparticles with reduced potential for aggregation. Synthesis and particle evaluation were performed in triplicate to confirm reproducibility of desired MSN synthesis. The XRD scan of the synthesized MSN displayed a sharp peak confirming the presence of ordered pores owing to a regular periodic variation of the electron density in the mesoporous silica nanoparticles (Ciesla and Schuth, 1999). The physicochemical properties of the synthesized MSN are summarized in table $\mathbf{1}$.

The particle size was further confirmed, and the morphology determined through scanning electron microscopy (SEM). This allowed for the surface of the nanosystem to be characterized and to ascertain if the morphology of the nanosystem was uniform. As seen in Figure 2, the particles displayed a uniform morphology, being oval in shape and the average size of the particles observed through the SEM concurred with the zeta analysis results, with an average approximate size of $108 \mathrm{~d} . n m$. (Figure 2). This uniformity was expected owing to the low PDI value which indicated an overall low polydispersity of the sample.

\section{Impregnation of MSN With CurNQ}

Following MSN synthesis and calcination, MSN was impregnated with CurNQ and Cur individually to create the nanosystems MSN_CurNQ and MSN_Cur, respectively. This was achieved through rapid mixing of the MSN and CurNQ in a small volume of methanol protected from light at room temperature for $48 \mathrm{~h}$, allowing for CurNQ to be impregnated in the pores of the MSN. Successful loading of MSN with CurNQ was confirmed through UV/Vis absorbance readings. The absorbance

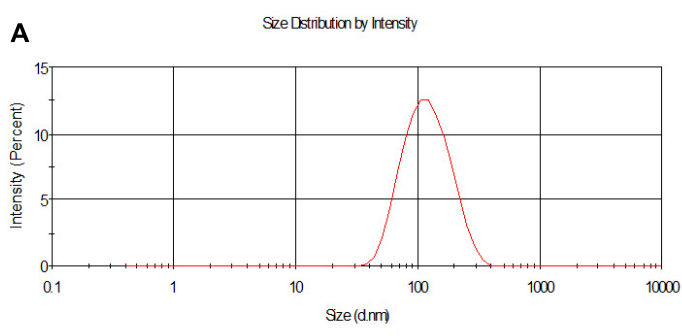

C

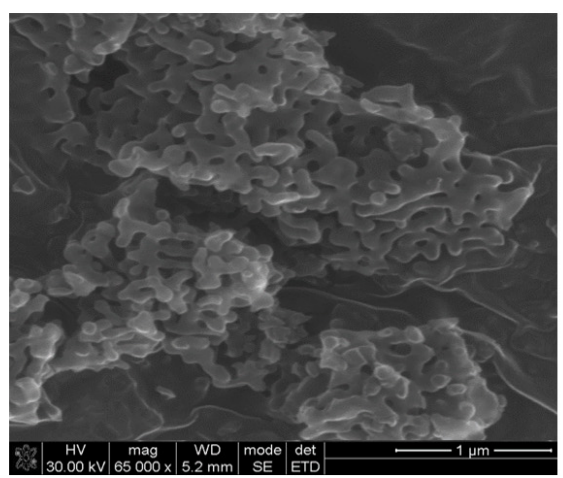

B Zeta Potentid Distribution

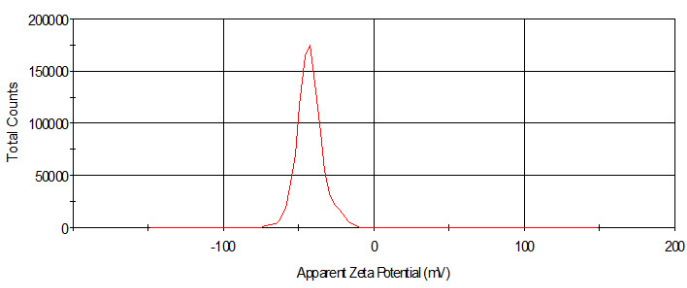

D

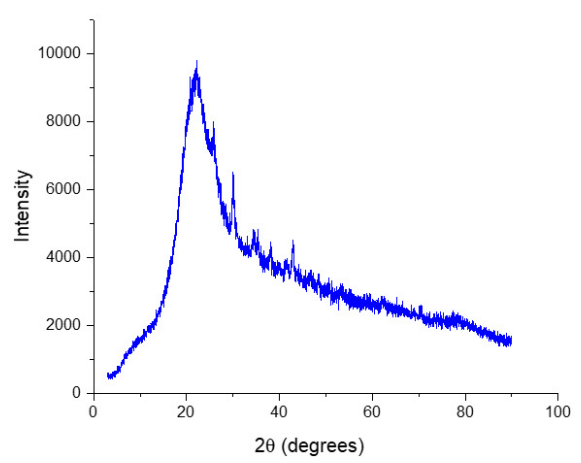

FIGURE 2 | Size, morphology and zeta potential analysis of synthesized MSN. (A) Size of MSM determined to be 108 d.nm through dynamic light scattering ( $N=3$ ) (B) Zeta potential of MSN obtained using the ZetaSizer and determined to be $-42 \mathrm{mV}(\mathrm{N}=3)$ (C) SEM microscopy image of the surface of MSN to evaluate surface morphology (D) XRD analysis of MSN.

TABLE 1 | Physicochemical properties of synthesized MSN.

\begin{tabular}{|c|c|c|c|c|c|c|}
\hline Parameter & Size (d.nm) & PDI & Zeta potential (mV) & BET Surface area $\left(\mathrm{m}^{2} / \mathrm{g}\right)$ & Pore volume $\mathrm{cm}^{3} / \mathrm{g}$ & Average pore size $\AA$ \\
\hline MSN & 108 & 0.150 & -42 & 30.0883 & 0.040908 & 54.3846 \\
\hline
\end{tabular}


readings were taken before, during and after the 48-h period. A decline in absorbance readings corresponded to the uptake of the drug into the pores of the nanoparticles, thereby leaving the solution and reducing the absorbance of the solution. This uptake was quantified, and the encapsulation efficiency was calculated. Moreover, to further confirm successful CurNQ encapsulation the size of the loaded nanoparticles was measured and compared to the empty nanoparticles. As expected, the size of the CurNQ loaded particles increased upon drug loading from an average of 108 to $214 \mathrm{~d}$.nm. This increase in size was owing to the pores of the particles being impregnated with the product. The above process was repeated for the loading of Cur into MSN, thereby acting as a control. The entrapment efficiencies were calculated to be 54,54 and $32,5 \%$ for CurNQ and Cur, respectively.

\section{Evaluation of $\mathrm{pH}-$ Responsiveness of MSN_CurNQ}

To support constant cell growth and proliferation, a cancer cell increases glycolysis and bypasses oxidative phosphorylation, this allows for the rapid production of ATP and for cellular proliferation to continue unabated (Warburg, 1925). This phenomenon is known as the "Warburg effect" and is a hallmark feature of oncogenesis. This increase in glycolysis induces the acidification of the ECM, owing to the production of lactate (Warburg, 1925). This results in the slight acidification of the tumor microenvironment and allows for the differentiation between cancerous and healthy tissue (Warburg, 1925). However, this overall $\mathrm{pH}$ reduction is slight. Therefore, exploiting this $\mathrm{pH}$ reduction to detect and target malignant cells would require a robust detection system which is highly $\mathrm{pH}$ sensitive and would elicit a large response to small fluctuations in $\mathrm{pH}$ levels. CurNQ displays $\mathrm{pH}$-responsive solubility within the $\mathrm{pH}$ change that occurs during malignant transformation and demonstrated a substantial increase in solubility with a shift in pH from 7.4 to 6.8 (Freidus et al., 2020). Moreover, CurNQ was demonstrated to have high fluorescent intensity thereby releasing a strong fluorescent signal once soluble. It was thought that this shift in solubility and concurrent fluorescence could act as a molecular switch allowing for potential tumor targeting and detection applications.

It was of interest to determine if this molecular switch occurred when CurNQ was loaded onto MSN and if this molecular switch could be exploited for cancer targeting; therefore, a release assay of CurNQ from the MSN was performed at $\mathrm{pH} 7.4$ and $\mathrm{pH}$ 6.8. This assay again included MSN_Cur acting as a comparison and allowed for the novelty of this $\mathrm{pH}$ switch to be highlighted. As seen in Figure 3, there was a large difference between the release profile of CurNQ at $\mathrm{pH} 7.4$ compared to at $\mathrm{pH} 6.8$, as well as a highly statistically significant different release profile between MSN_CurNQ and MSN_Cur $(p=0.0001)$. The release of CurNQ at $\mathrm{pH} 6.8$ is just less than double its release at $\mathrm{pH}$ 7.4. At physiological $\mathrm{pH}$ (7.4), there was only $10 \%$ release within the first $12 \mathrm{~h}$, with it reaching $20 \%$ after $24 \mathrm{~h}$ and peaking at $31,5 \%$ at $96 \mathrm{~h}$.

This was in stark contrast to the release profile of MSN_CurNQ at $\mathrm{pH}$ 6.8. With this small reduction in $\mathrm{pH}$, the

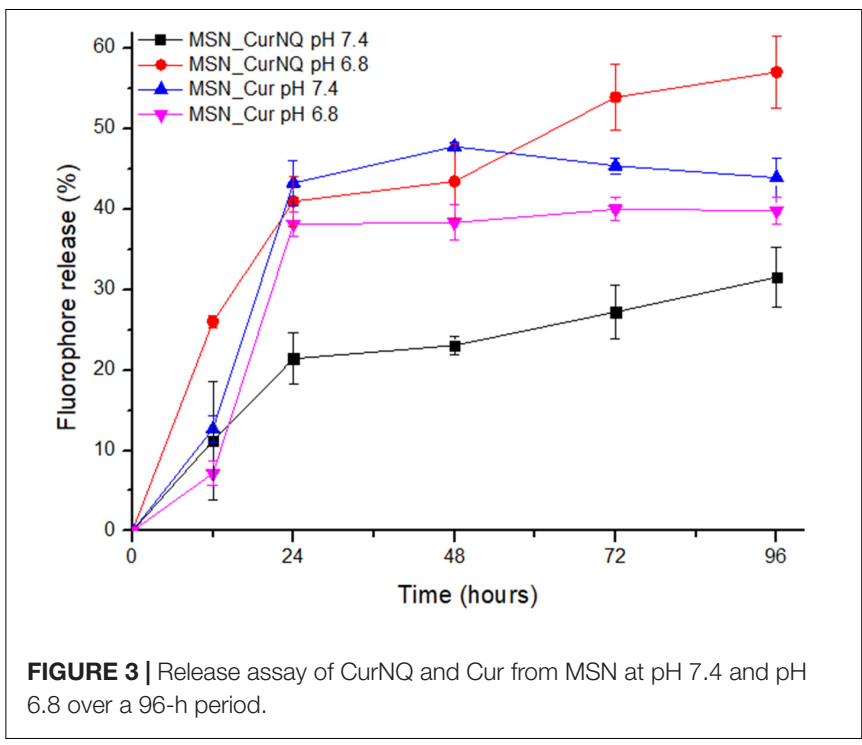

novel nanosystem released $43,45 \%$ after $48 \mathrm{~h}$, with the release peaking at $57 \%$ at $96 \mathrm{~h}$. This equates to a $25,5 \%$ increase in release occurring in response to a 0.6 reduction in $\mathrm{pH}$. Consequently, the release of CurNQ at $\mathrm{pH} 6.8$ was almost double the release at $\mathrm{pH}$ 7.4. This difference in release between the two $\mathrm{pH}$ values was highly statistically significant with a corresponding $\mathrm{p}$-value of 0.000 . This highly significant $p$-value confirms the effects of $\mathrm{pH}$ on the release of CurNQ from the MSN, verifying the $\mathrm{pH}$ responsivity and specificity of the nanosystem. Moreover, this highly statistically significant $p$-value emphasizes the robust nature of CurNQ pH-responsivity. Clearly, the change in $\mathrm{pH}$ associated with malignant transformation acts as a solubility switch for CurNQ. This $\mathrm{pH}$ specificity was not observed for MSN_Cur $(p=0.31)$, and notably MSN_Cur exhibited a slightly greater release at physiological $\mathrm{pH}$, thereby indicating its nonspecific $\mathrm{pH}$ solubility and its inability to act as a tumor targeting agent.

The release profile aligns with the saturation solubility results presented in our previous research (Freidus et al., 2020). At pH 7.4 CurNQ's saturation solubility was determined to be $11.5 \mu \mathrm{M}$, compared to $20.7 \mu \mathrm{M}$ at $\mathrm{pH}$ 6.8. This drastic increase in solubility in response to small fluctuations in $\mathrm{pH}$ levels would allow CurNQ to act as a cancer targeting molecule through a $\mathrm{pH}$ specific solubility switch. Figure 3 confirms that this solubility switch occurs once CurNQ was loaded into MSN as the release of CurNQ from the MSN occurred in a $\mathrm{pH}$ dependent manner.

The synthesis of CurNQ saw the addition of two quinone moieties and the substantial $\mathrm{pH}$ dependent release profile difference between Cur and CurNQ can be attributed to the addition of these quinone moieties. Quinones undergo a reversible two-electron redox reaction and the corresponding redox potential is strongly $\mathrm{pH}$ dependent. At acidic $\mathrm{pH}$, reduction occurs and is a single step two-electron two-proton process and this reduction forms a hydroquinone and this hydroquinone form has a greater solubility than the quinone form. Thus, this redox reaction accounts for the switch in solubility and the $\mathrm{pH}$ dependent release. 
Furthermore, Figure 3 demonstrated the increase of release of CurNQ over a period of $96 \mathrm{~h}$ thereby demonstrating sustained release of CurNQ from the MSN. This sustained release is imperative to allow for the fluorescent detection of a tumor and to offer effective treatment upon release, owing to sustained exposure to the anti-cancer agent. Again, this sustained release is not evident in MSN_Cur, as after $48 \mathrm{~h}$, there is no longer further release of Cur, further highlighting the novelty and applicability of the novel nanosystem.

Targeting the slightly acidic tumor microenvironment for tumor targeting, detection and specific drug release has been of great interest for cancer theranostic applications. Several strategies have been investigated by several researchers to achieve this. Such strategies have included the modification of nanoparticles whereby their surface was modified with a $\mathrm{pH}$ sensitive compound such as glycol chitosan or through the introduction of protonatable groups. Other avenues include using polymers whereby acid labile bonds are introduced or through an amphiphilic polymer triggered prodrug. Other researchers have used calcium carbonate nanocrystals loaded with cancer drugs that disassociate at the tumor microenvironment, thereby releasing the drug at the desired location (Kanamala et al., 2016; Gulzar et al., 2019). Moreover, many of these strategies utilize the acidic $\mathrm{pH}$ of the exosome or lysosome that allows for drug uptake, as the $\mathrm{pH}$ in the exosome or lysosome is substantially different to the $\mathrm{pH}$ of the cytosol or the microenvironment, thus $\mathrm{pH}$ differentiation is easier to achieve compared to the highly sensitive system that is required to detect the change to a slightly acidic microenvironment that accompanies malignancy (Kanamala et al., 2016; Gulzar et al., 2019).

The realization of $\mathrm{pH}$-responsiveness in this study differs to all the strategies mentioned above. To best of our knowledge we are not aware of any active compound that intrinsically offers robust $\mathrm{pH}$ sensitivity without the addition of nanomaterials or $\mathrm{pH}$ sensitive groups. The simplicity of the $\mathrm{pH}$-responsive solubility of the active compound is unique and is highly novel, especially owing to the sensitivity and alignment to the $\mathrm{pH}$ change that occurs during malignancy. Not only is the molecule highly $\mathrm{pH}$ specific which is rare, but it offers this specificity at the exact change in $\mathrm{pH}$ that is stimulated in response to oncogenesis. Clearly, this is an exceptional finding and can be exploited for tumor targeting and detection applications.

\section{Evaluating the Innate Fluorescence of MSN_CurNQ and Its Detection Capabilities}

The need for novel fluorophores for imaging applications is immense and in vivo imaging and detection devices can be applied in numerous and varied applications. The synthesis of a fluorescent compound that is ideal for imaging applications is a large area of research (Freidus et al., 2018). Here, the aim was to extend previous research in the synthesis of a novel fluorescent compound that possesses additional theranostic attributes. The considerations when designing a new fluorescent compound that is ideal for imaging applications is immense.
An ideal fluorophore for imaging applications should have an appropriate background to target ratios, high specificity, appropriate absorption and emission wavelengths to avoid tissue autofluorescence, appropriate solubility and fluorescence stability, exhibit cell permeability and have a long fluorescent lifetime (Freidus et al., 2018).

The fluorescent properties of CurNQ were determined through obtaining the absorption and fluorescence spectra, which were measured and recorded on the Shimadzu UV-1800 spectrophotometer and Shimadzu RF-6000 spectrofluorophotometer (Maryland, United States) respectively. The absorption peak was determined to be $429 \mathrm{~nm}$, the excitation peak $595 \mathrm{~nm}$ and the emission peak at $670 \mathrm{~nm}$.

CurNQ has a high fluorescence intensity and its $\mathrm{pH}-$ responsive solubility would allow it to release a fluorescent signal at the $\mathrm{pH}$ of the tumor microenvironment. However, without the loading of CurNQ onto a nanoparticle, the fluorescence of CurNQ cannot properly be traced and visualized, as generalized fluorescence is difficult to pinpoint in vitro and in vivo. Therefore, the loading of CurNQ onto a delivery vehicle is crucial to exploit its fluorescent capabilities for detection applications. In order to explore these fluorescent capabilities and to determine if MSN_CurNQ can be used for detection and imaging applications, confocal microscopy utilizing super resolution Airyscan technology and fluorescent microscopy on the CELENA ${ }^{\circledR}$ X High Content Imaging System were performed.

These assays were used to verify fluorescence and to determine whether the loaded nanoparticles could be accurately detected and visualized using the innate fluorescent properties of CurNQ; moreover, cellular uptake could be evaluated. Cellular uptake is important as for an active compound to exert its desired effect, it is required to enter the cell. The ovarian cancer cell line OVCAR-5 was utilized for this assay.

Cells were incubated with MSN_CurNQ for $24 \mathrm{~h}$, to allow for cellular uptake to occur. Subsequently, cells were fixed in formaldehyde and stained with DAPI. The cells were then viewed under the Zeiss LSM 780 confocal microscope (Figures 4, 5) as well as the CELENA ${ }^{\circledR} \mathrm{X}$ High Content Imaging System (Figure 6).

As seen in the microscopy images, MSN_CurNQ displayed clear, distinctive, high intensity fluorescence. The fluorescence was distinctive enough to pinpoint small clusters of nanoparticles, with almost no fluorescent blur. Moreover, a high target to background ratio with low background fluorescence and high fluorescence specificity was observe. This is a crucial requirement for a fluorophore effective for in vivo imaging. Hence, this distinctive and precise fluorescence with low background poses MSN_CurNQ as a potentially powerful imaging agent. Cell permeability is critical to allow for internal cellular imaging, as well as required for therapeutic applications; as seen in the above images, MSN_CurNQ exhibited cell permeability, and was evenly distributed throughout the cytoplasm. No loss of fluorescent intensity was observed after repetitive imaging over time using the same sample, therefore MSN_CurNQ has a long fluorescent lifetime, has stable fluorescence as was not easily photodegraded or photobleached. These fluorescent properties potentially offer the 

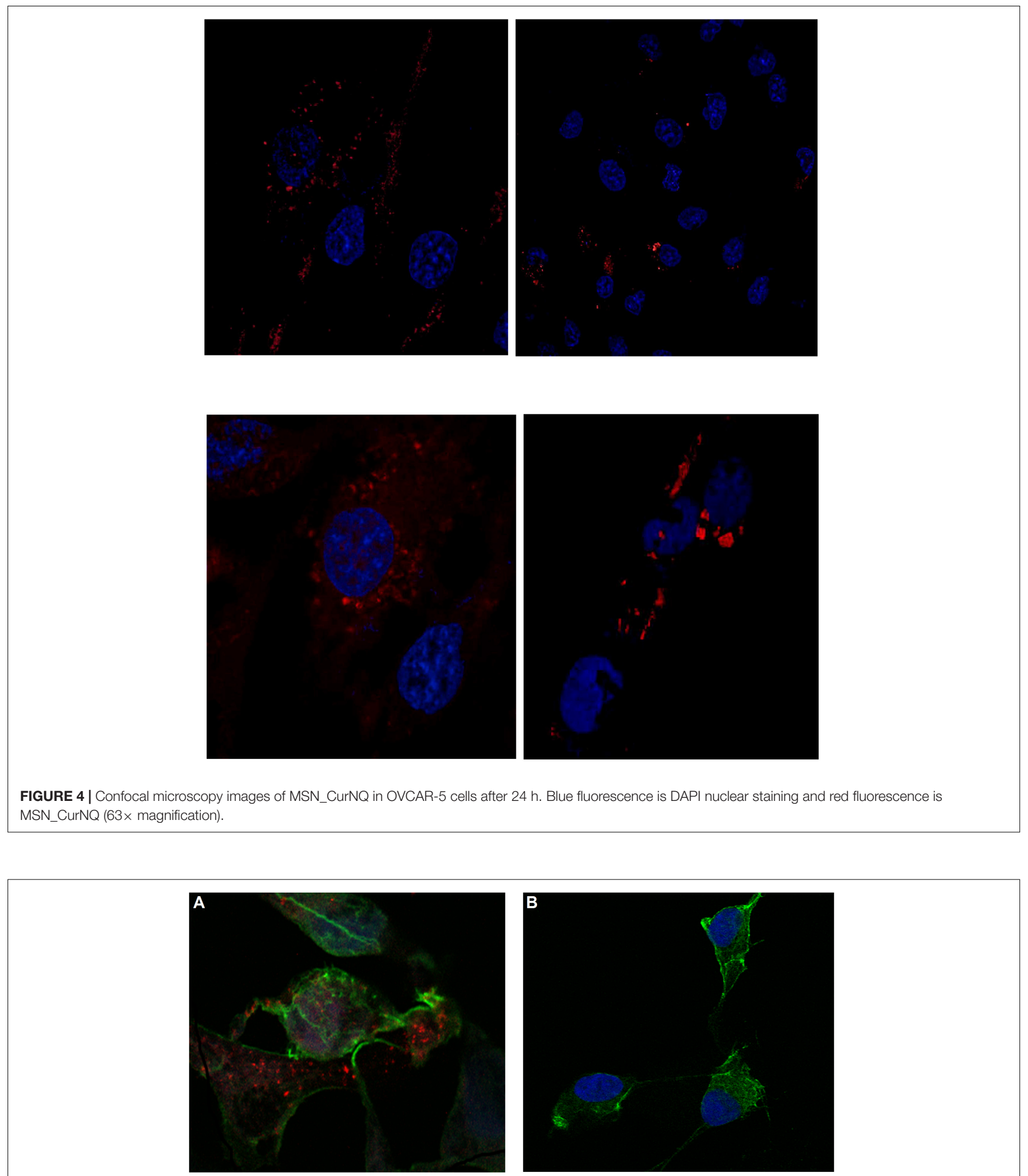

FIGURE 5 | Confocal microscopy images of MSN_CurNQ in OVCAR-5 cells after $24 \mathrm{~h}$ (63× magnification). These confocal microscopy images demonstrate that CurNQ is required to be loaded onto a delivery vehicle to allow for fluorescence detection and tracking. (A) OVCAR-5 cells treat with MSN_CurNQ for $24 \mathrm{~h}$ (63x magnification) (B) OVCAR-5 cells treated with CurNQ for $24 \mathrm{~h}$ (no MSN). Blue fluorescence is DAPI nuclear staining, green fluorescence is Phalloidin-Atto 488 actin stain and red fluorescence is MSN_CurNQ (63× magnification). 


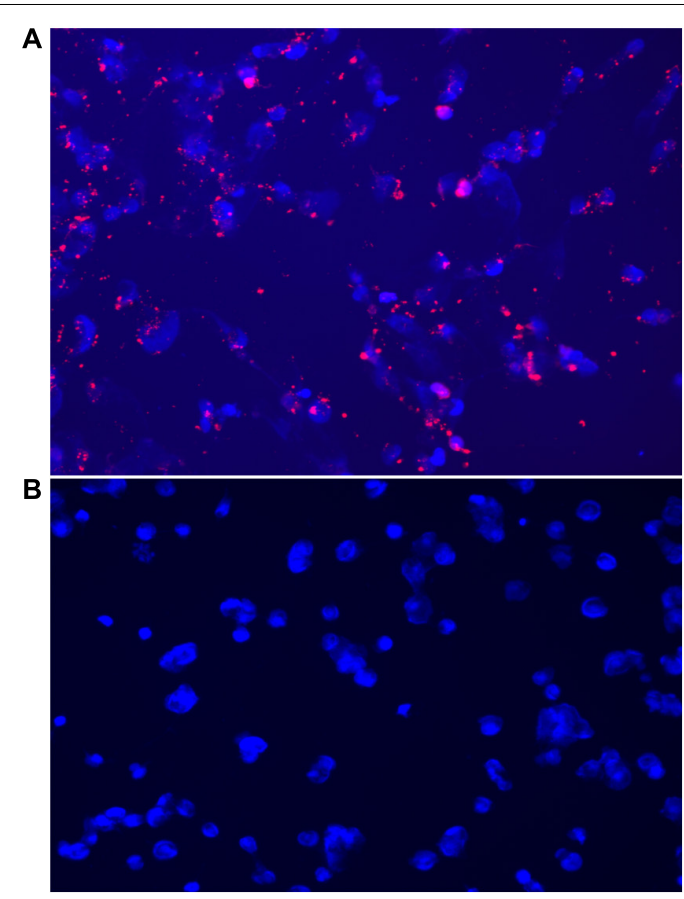

FIGURE 6 | Fluorescent microscopy images acquired on the CELENA ${ }^{\oplus} X$ High Content Imaging System of (A) OVCAR-5 cells incubated with MSN_CurNQ for $24 \mathrm{~h}$ (20x magnification). Blue fluorescence is DAPI nuclear staining and red fluorescence is MSN_CurNQ. (B) Cells given no treatment (control). No red fluorescence is present in the control group, confirming fluorescence is from MSN_CurNQ.

opportunity to extend the use of MSN_CurNQ for molecular imaging applications.

Moreover, the fluorescent images demonstrate that there was cellular uptake of MSN_CurNQ, but not nuclear uptake. The nanoparticles can be observed to cluster around the nucleus, yet they do not enter the nucleus. Cellular uptake is of great importance, as for a drug to elicit its response, it is required to enter the cell. Clearly, cellular uptake occurs allowing for MSN_CurNQ to elicit its potential anti-cancer effects. Cellular uptake may occur through endocytosis or membrane permeation; however; the method of uptake cannot be determined through the above microscopy images.

As discussed above, it was expected that the inclusion of a nanosystem would be required to realize the potential detection and imaging applications of CurNQ. Therefore, it was of interest to visualize cells treated with the loaded nanosystem MSN_CurNQ and CurNQ treatment alone. As seen in Figure 5, no red fluorescence is observable in cells treated with CurNQ alone, this indicates that CurNQ needs to be loaded onto a nanoplatform for detection capabilities to be realized.

One of the main objectives of this research as to synthesize a molecule with appropriate fluorescent properties to allow for detection and imaging applications. Cur cannot be used for detection or imaging applications owing to its short fluorescent lifetime and its low quantum yield. Furthermore, it undergoes rapid photo bleaching and has a low quantum yield and fluorescent intensity in aqueous solution (Priyadarsini, 2009). Therefore, it was a challenge to improve these fluorescent properties with Cur's core structure being present in CurNQ and to develop a fluorescent molecule having suitable properties for fluorescence detection applications. Cur has an excitation and emission spectra of $450 \mathrm{~nm}$ and $550 \mathrm{~nm}$ and these wavelengths would induce tissue autofluorescence if used in in vivo applications, therefore a longer excitation wavelength is required to avoid autofluorescence. The synthesis of CurNQ resulted in the excitation and emission wavelengths being significantly red shifted compared to Cur. CurNQ had an excitation and emission wavelength of 595 and $670 \mathrm{~nm}$, respectively, this equates to a red shift of $145 \mathrm{~nm}$. This is noteworthy, as excitation at $595 \mathrm{~nm}$ would substantially reduce tissue autofluorescence and would allow for effective in vivo imaging. The stokes shift was determined to be $75 \mathrm{~nm}$, this significant gap between the excitation and emission spectra would reduce fluorescence selfabsorbance thereby allowing for in vivo imaging application (Qi et al., 2018).

The microscopy images demonstrated MSN_CurNQ to have highly precise, distinctive and high intensity fluorescence. Moreover, a high target to background ratio, low fluorescent blur, a long fluorescent lifetime and fluorescent stability indicate MSN_CurNQ to be a potentially powerful diagnostic and imaging agent.

\section{Evaluating the Chemotherapeutic Properties of MSN_CurNQ Through High Content Imaging}

High content imaging is an advanced technique that combines high throughput techniques and fluorescent microscopy to obtain quantitative data from complex biological systems (Zanella et al., 2010). It is a robust method utilizing an automated microscope that allows for rapid, high content image acquisition and analysis (Zock, 2009). Multiple independent measurements can be collected from a single cell or a single cell population. Herein, this advanced technique was employed using the CELENA ${ }^{\circledR} \mathrm{X}$ High Content Imaging System. The high content imager was used to measure cell viability in response to MSN_CurNQ treatment; moreover, the number of nanoparticles were counted, enabling the evaluation of the effect of concentration on cell viability.

As seen in Figure 7, there was a dose dependent response to MSN_CurNQ treatment. With an increasing count of loaded MSN, there was a decrease in cell count, occurring in an inversely proportional manner. Therefore, treatment with MSN_CurNQ resulted in a decrease in cell viability. The visual representation with concurrent qualitative data acquired from the high content imager makes the high content imaging system a powerful tool in evaluating the anti-cancer properties of a particular system and in the drug discovery process. These results suggest the MSN_CurNQ has potential to act as a chemotherapeutic agent.

\section{In vitro Cytotoxicity Assay-MTT}

It was of interest to investigate the cytotoxic nature of MSN_CurNQ on a variety of cancer cell types. OVCAR-5 cells 


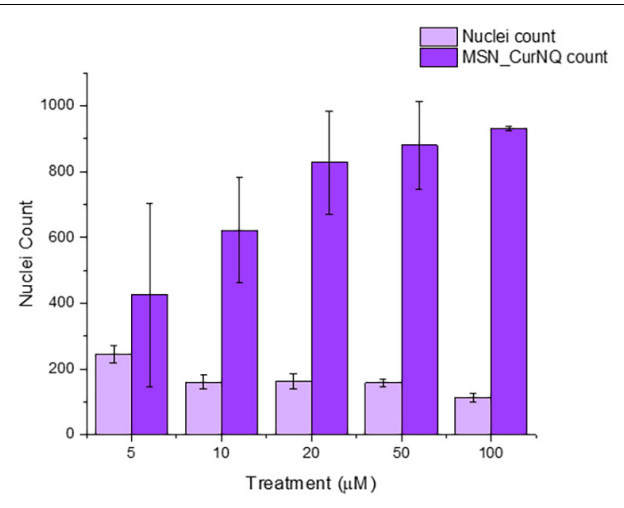

FIGURE 7 | Nuclei count, and MSN count of OVCAR- 5 cells treated with varying concentrations of MSN_CurNQ for 24-h. Nuclei and MSN count were quantitatively obtained using a high content imaging system.

were used as the model cell type when investigating the anticancer effects of CurNQ, thus it was necessary that this cell line was carried through to the research on MSN_CurNQ. The inclusion of MCF-7 and HeLa aimed to expand the area of investigation to other female cancer types. Moreover, the additional of varied cancer cell types aimed to give a broad investigational approach to the anticancer effects of MSN_CurNQ on account of the novelty of the nanosystem.

To this end, MTT assays were performed on 6 cancer cell lines and one non-malignant fibroblast cell line. The purpose of these MTT assays were two-fold. Firstly, it was crucial to determine if CurNQ would induce its previously observed potent anti-cancer effects whilst loaded onto the MSN delivery vehicle and secondly to evaluate if the cytotoxicity of CurNQ was translatable to a variety of cancer types.

As seen in Figures 8, the cytotoxicity of MSN_CurNQ and MSN_Cur depended greatly on the cancer cell type. Certain cancer cells such as CACO-2 and OVCAR-5 exhibited large reductions in cell viability, whereas other cancer cell lines such as HeLa experienced low overall toxicity in response to the loaded nanoparticles. Concentration administered and incubation time both affected overall cytotoxicity; however, cancer cell type was the largest determining factor.

Cell viability was reduced to the greatest extent in the OVCAR5 and CACO- 2 cell lines. Cell viability dropped substantially after $48 \mathrm{~h}$ of incubation for both cell lines (Figure 9). This aligns with Figure 3 which demonstrated the prolonged release of CurNQ from the nanoparticles over a period of $96 \mathrm{~h}$, thus the reduction in cell viability occurred once CurNQ was released. Release of CurNQ from the MSN increased drastically after $24 \mathrm{~h}$ (Figure 3), after which it would be able to exert its effects culminating in the decline in cell viability at $48 \mathrm{~h}$. This sustained release is ideal for anti-cancer mechanisms, as it first allows for the nanoparticles to accumulate in the tumor prior to the complete release of the active compound. Additionally, this sustained release would allow for a longer detection window, in respect to the nanosystem's proposed detection applications.

Although cell viability was reduced to the greatest extent at $48 \mathrm{~h}$, at the 24 -h incubation period, $400 \mathrm{ng} / \mathrm{mL}$ of MSN_CurNQ induced a reduction in cell viability to $28,5 \%$ in OVCAR-5 cells. This shows that this high concentration of CurNQ induced immediate cell death and this same response of immediate cell death occurred in the CACO-2 cell line. This suggests that enough CurNQ was released to induce cell death after $24 \mathrm{~h}$ on account of the high concentration administered. In the OVCAR5 cell line, time was the most significant factor in the reduction of cell viability, as after $48 \mathrm{~h}$, all treatment concentrations induced a reduction in cell viability to below $50 \%$. However, cell recovery was observed after $72 \mathrm{~h}$ in cells receiving treatments of less than $200 \mathrm{ng} / \mathrm{mL}$ of MSN_CurNQ, whereas cell viability remained low after $72 \mathrm{~h}$ in cells that received $200 \mathrm{ng} / \mathrm{mL}$ or higher of MSN_CurNQ. This same pattern of cell recovery in response to low doses of the nanosystem was observed in CACO2 cells, with the recovery being greater; but again, treatment with $200 \mathrm{ng} / \mathrm{mL}$ or greater induced sustained reductions in cell viability. Therefore, higher concentrations of treatment are required to induce lasting cell death.

MCF-7 and CHLA cell lines also demonstrated a reduction in cell viability but this reduction was slightly less compared to OVCAR-5 and CACO-2, yet cell viability was still reduced to below 50\%, hence MSN_CurNQ shows promise as a broad chemotherapeutic agent that has the ability to act against a variety of cancer types. Interestingly, the MCF-7 and CHLA cell lines experienced their greatest reduction in cell viability after $24 \mathrm{~h}$, differing from the response observed for OVCAR-5 and CACO-2 cell lines. While the release from MSN peaks after $96 \mathrm{~h}$, there is not a large difference in release between 24 and $48 \mathrm{~h}$ on account of the sustained release from the nanoparticles. The MCF-7 and CHLA cell lines had an immediate response to treatment, with cell viability reducing to below $50 \%$ in cells receiving $200 \mathrm{ng} / \mathrm{mL}$ or higher of MSN_CurNQ. However, MCF-7 and CHLA cells recovered subsequent to their initial reduction in cell viability, and this also occurred in cells receiving the highest concentration of MSN_CurNQ; yet cells do not recover to full viability suggesting treatment was still effective in reducing the overall cell population. Although cell viability reduction was not as high as OVCAR-5 and CACO-2, the reduction in cell viability of MCF-7 and CHLA was statistically significantly different to the control cell line 3T3 $(p=0.000)$, indicating MSN_CurNQ treatment does result in a statistically significant reduction in cell viability to a variety of cancer types. Accordingly, it is recommended that in an in vivo setting, that a treatment regime is instigated, whereby a patient would receive several doses of the nanosystem, as to ensure malignant cell recovery does not occur.

There was a slight reduction in cell viability in HT-29 cells, cell viability was reduced to around 70\%; and very low reductions in cell viability were observed for the HeLa cell line. This small reduction in cell viability precludes MSN_CurNQ's chemotherapeutic application for these cancer types, as a greater reduction in cell viability are required for a system to be considered to have chemotherapeutic applications. On the contrary, MSN_CurNQ can still be applied as a detection and targeting system in these cancers. Owing to the multifaceted nature of MSN_CurNQ, its applications are not limited to a select few cancer types. Indeed, further treatment options would be required for cancer types that are not adequately treated by 

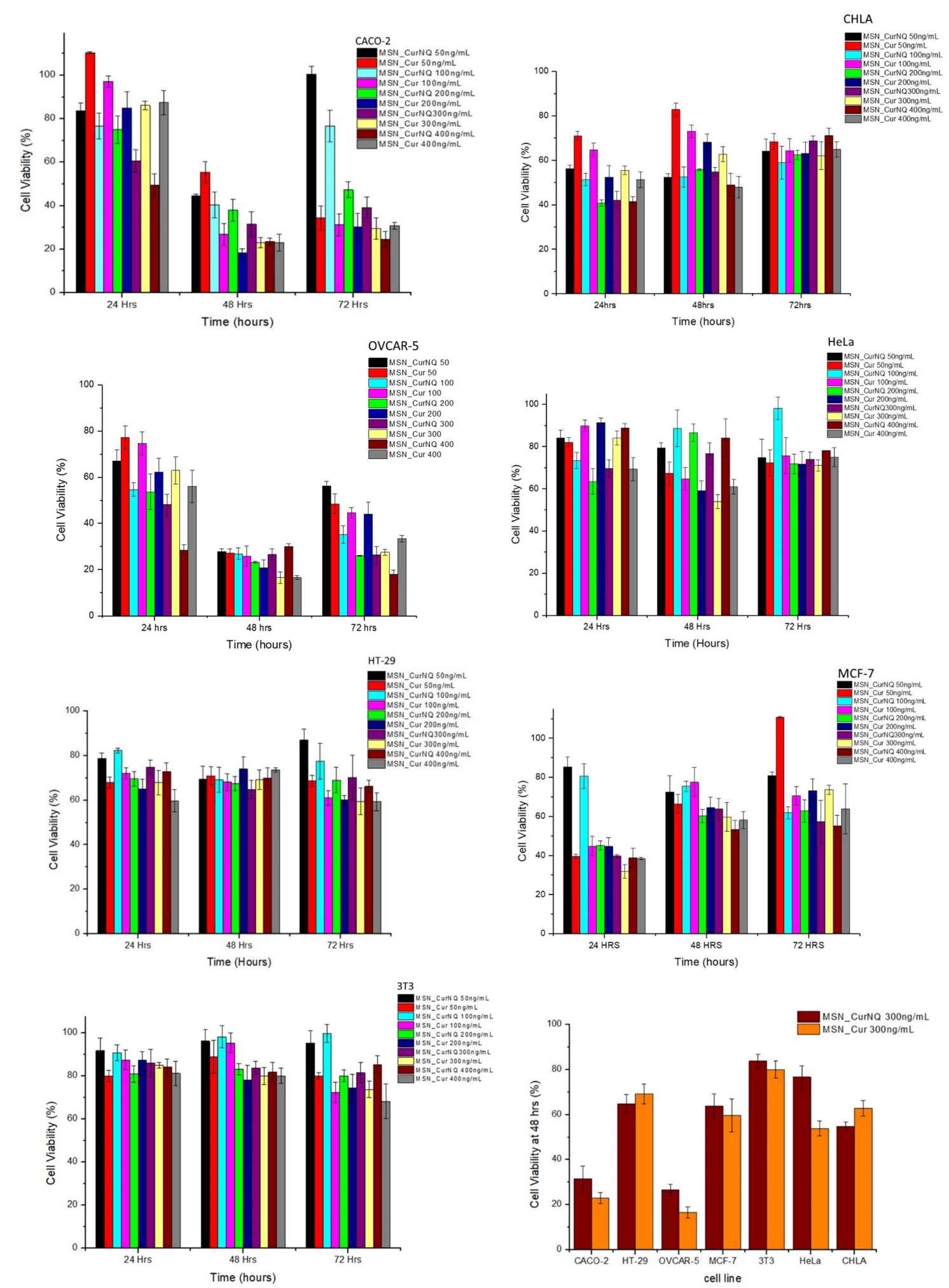

FIGURE 8 | Cytotoxicity of MSN_CurNQ and MSN_Cur to CHLA, CACO-2, OVCAR-5, HeLa, HT-29, MCF-7, and 3T3 cell lines. MSN_CurNQ and MSN_Cur treatments were administered at a range of concentrations over a 72-h period and the reductions in cell viability were determined through an MTT assay. Statistically significant reductions in cell viability toward MSN_CurNQ treatment compared to the control cell line were observed for cell lines OVCAR-5, CACO-2, CHLA, HT-29, and MCF-7 cell lines. 

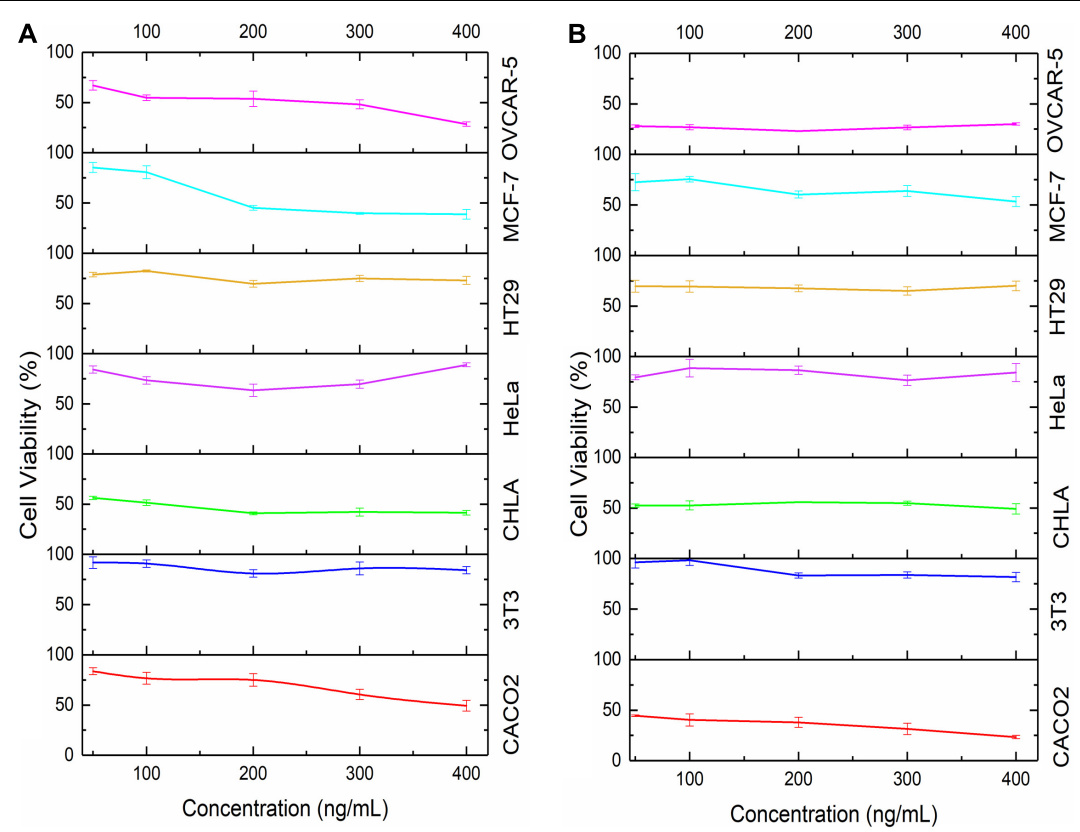

FIGURE 9 | Cell viability in response to concentration of MSN_CurNQ after (A) $24 \mathrm{~h}$ and (B) $48 \mathrm{~h}$ in six cancer cell lines and in 1 healthy fibroblast cell line.

MSN_CurNQ; however, its application as a first line detection and targeting system is applicable.

Statistical analyses were performed to ascertain the effect of MSN_CurNQ and MSN_Cur on a variety of cancer cell types and this reduction was compared to the healthy fibroblast cell line 3T3. Statistical analyses were performed using Stata/IC 15.1 statistical software. The cell line was indeed a statistically significant factor for the reduction of cell viability $(p=0.0000)$. Administered concentration was also a statistically significant factor in cell viability reduction $(p=0.0000)$. OVCAR- 5 cells were the most responsive to MSN_CurNQ treatment, and induced cell death was significantly different to each other cell line tested (HeLa, CHLA, MCF-7, HT-29, and 3T3 $p=0.000$; CACO$2 p=0.035)$. Importantly, there was a significant difference in the reduction in cell viability of the healthy cell line $3 \mathrm{~T} 3$ to all other cancer cell lines investigated, barring HeLa cells (OVCAR-5, CACO-2, MCF-7, CHLA $p=0.000$, HT-29 $p=0.004$; HeLa $p=0.378$ ). This means that MSN_CurNQ reduced cancer cell viability to a greater extent than it did to a healthy fibroblast cell line. The $p$-values of 0.000 and 0.004 indicate a high degree of statistically significance and verify the selective toxicity of MSN_CurNQ to cancer cells, meaning MSN_CurNQ has a higher potency toward cancerous cells than to healthy fibroblast cells. This selective toxicity further extends the cancer targeting capabilities of the nanosystem and further cements its novelty and applicability in the field of cancer theranostics.

The obtained results indicate a high concentration of MSN_CurNQ is required for effective and sustained reductions in cell viability. This high required dosage would not be a limiting factor to successful treatment on account of the observed cancer selective toxicity. Cell viability remained above $80 \%$ for the healthy fibroblast cell line 3T3, after treatment with $400 \mathrm{mg} / \mathrm{mL}$ of MSN_CurNQ, thereby indicating that MSN_CurNQ is non-toxic to these healthy fibroblast cells. This suggests that it would be safe to administer high concentrations of MSN_CurNQ to patients, as this should induce large reductions in cancer cell viability and a very low reduction to healthy cell viability.

Indeed, this research demonstrated that CurNQ requires to be loaded onto a nanoplatform to allow for all its theranostic capabilities to be realized. MSN_CurNQ thereby offers a threepronged approach for cancer targeting namely its $\mathrm{pH}$-responsive solubility, the EPR effect and its selective toxicity toward cancer cells. This makes MSN_CurNQ a highly advanced and novel nanosystem for cancer theranostics.

\section{CONCLUSION}

The loading of CurNQ onto the MSN platform aimed to allow for enhanced delivery and enable in vitro and in vivo imaging and detection. To this end, MSN were synthesized and were impregnated with CurNQ to form the novel nanosystem MSN_CurNQ. CurNQ demonstrated $\mathrm{pH}$ responsive release from MSN, specific to the tumor microenvironment whereby after 96 h $31.5 \%$ of CurNQ was released at pH 7.4 compared to 57\% release at $\mathrm{pH} 6.8$, which thereby may allow for tumor targeting applications owing to $\mathrm{pH}$ responsivity. Moreover, MSN_CurNQ exhibited high intense, clear and distinctive innate fluorescence enabling detection and possible imaging applications. Moreover, MSN_CurNQ induced cytotoxicity culminating in a reduction to below 50\% cell viability in OVCAR-5, CACO-2, CHLA, and MCF-7 cell lines thereby demonstrating its chemotherapeutic potential to a variety of cancer cell types. Furthermore, 
MSN_CurNQ treatment did not induce cytotoxicity to the healthy fibroblast cell line 3T3, demonstrating cancer selective toxicity, furthering the nanosystem's novelty and potential applications. Clearly, the novel nanosystem MSN_CurNQ has potential applications in cancer theranostics.

\section{DATA AVAILABILITY STATEMENT}

The raw data supporting the conclusions of this article will be made available by the authors, without undue reservation.

\section{AUTHOR CONTRIBUTIONS}

PK, PP, and YC conceptualized the research. LF, TM, and PP contributed to methods and experimentation. LF wrote the first

\section{REFERENCES}

Alyassin, Y., Sayed, E. G., Mehta, P., Ruparelia, K., Arshad, M. S., Rasekhet, M., et al. (2020). Application of mesoporous silica nanoparticles as drug delivery carriers for chemotherapeutic agents. Drug Discov. Today. 25, 1513-1520. doi: 10.1016/j.drudis.2020.06.006

Argyo, C., Weiss, V., Bra, C., and Bein, T. (2013). Multifunctional mesoporous silica nanoparticles as a universal platform for drug delivery. Chem. Mater. 26, 435-451. doi: 10.1021/cm402592t

Baeza, A., Manzano, M., Colilla, M., and Vallet-Regí, M. (2016). Recent advances in mesoporous silica nanoparticles for antitumor therapy: our contribution. Biomater. Sci. 4, 803-813. doi: 10.1039/c6bm00039h

Bawa, P., Pillay, P., Choonara, Y. E., du Toit, L. C., Ndesendo, V. M., and Kumar, P. (2011). A composite polyelectrolytic matrix for controlled oral drug delivery. AAPS PharmSciTech. 12, 227-238. doi: 10.1208/s12249-010-9576-8

Bernardos, A., and Kouřimská, L. (2013). Applications of mesoporous silica materials in food - a review. Czech J. Food Sci. 31, 99-107. doi: 10.17221/240/ 2012-cjfs

Bray, F., Ferlay, J., Soerjomataram, I., Siegel, R. L., Torre, L. A., and Jemal, A. (2018). Global cancer statistics 2018: GLOBOCAN estimates of incidence and mortality worldwide for 36 cancers in 185 countries. CA Cancer J. Clin. 68, 394-424. doi: 10.3322/caac. 21492

Ciesla, U., and Schuth, F. (1999). Ordered mesoporous materials. Micropor. Mesopor. Mater. 27, 131-149. doi: 10.1016/s1387-1811(98)00249-2

Freidus, L. G., Kumar, P., Marimuthu, T., Pradeep, P., Pillay, V., and Choonara, Y. E. (2020). Synthesis and properties of CurNQ for the theranostic application in ovarian cancer intervention. Molecules 25:4471. doi: 10.3390/ molecules25194471

Freidus, L. G., Pradeep, P., Kumar, P., Choonara, Y. E., and Pillay, V. (2018). Alternative fluorophores designed for advanced molecular imaging. Drug Discov. Today 23, 115-133. doi: 10.1016/j.drudis.2017. 09.008

Gulzar, A., Xu, J., Wang, C., He, F., Yang, D., Gai, S., et al. (2019). Tumour microenvironment responsive nanoconstructs for cancer theranostic. Nano Today 26, 16-56. doi: 10.1016/j.nantod.2019.03.007

Haider, M., Abdin, S. M., Kamal, L., and Orive, G. (2020). Nanostructured lipid carriers for delivery of chemotherapeutics: a review. Pharmaceutics 12:288. doi: 10.3390/pharmaceutics12030288

Hu, J. J., Liu, L. H., Li, Z. Y., Zhuo, R. X., and Zhang, X. Z. (2016). MMPresponsive theranostic nanoplatform based on mesoporous silica nanoparticles for tumor imaging and targeted drug delivery. J. Mater. Chem. B 4, 1932-1940. doi: $10.1039 / \mathrm{c} 5$ tb02490k

Ivey, J. I., Bonakdar, M., Kanitkar, A., Davalos, R. V., and Verbridge, S. S. (2016). Improving cancer therapies by targeting the physical and chemical hallmarks of the tumor microenvironment. Cancer Lett. 380, 330-339. doi: 10.1016/j.canlet. 2015.12.019

Kanamala, M., Wilson, W. R., Yang, M., Palmer, B. D., and Wu, Z. (2016). Mechanisms and biomaterials in $\mathrm{pH}$-responsive tumour targeted drug draft. PK and YC reviewed and revised the manuscript. All authors approved the final draft.

\section{FUNDING}

This work was supported by the National Research Foundation (NRF) of South Africa.

\section{ACKNOWLEDGMENTS}

Viness Pillay, who passed away in July 2020, is hereby kindly acknowledged and remembered for his contributions to the conceptualization of this work. Wits Life Science Imaging Facility for the use of their Confocal Microscope.

delivery: a review. Biomaterials 85, 152-167. doi: 10.1016/j.biomaterials.2016. 01.061

Liu, J., Luo, Z., Zhang, J., Luo, T., Zhou, J., Zhao, X., et al. (2016). Hollow mesoporous silica nanoparticles facilitated drug delivery via cascade $\mathrm{pH}$ stimuli in tumor microenvironment for tumor therapy. Biomaterials 83, 51-65. doi: 10.1016/j.biomaterials.2016.01.008

Mai, W. X., and Meng, H. (2013). Mesoporous silica nanoparticles: a multifunctional nano therapeutic system. Integr. Biol. 5, 19-28. doi: 10.1039/ c2ib20137b

Ma’mani, L., Nikzad, S., and Kheiri-manjili, H. (2014). Chemistry curcumin-loaded guanidine functionalized PEGylated I3ad mesoporous silica nanoparticles KIT6: practical strategy for the breast cancer therapy. Eur. J. Med. Chem. 83, 646-654. doi: 10.1016/j.ejmech.2014.06.069

Maruthappu, M., Head, M. G., Zhou, C. D., Gilbert, B. J., El-Harasis, M. A., Raine, R., et al. (2017). Investments in cancer research awarded to UK institutions and the global burden of cancer 2000-2013: a systematic analysis. BMJ Open 7:e013936. doi: 10.1136/bmjopen-2016-013936

Moreira, A. F., Dias, D. R., and Correia, I. J. (2016). Stimuli-responsive mesoporous silica nanoparticles for cancer therapy: a review. Micropor. Mesopor. Mater. 236, 141-157. doi: 10.1016/j.micromeso.2016.08.038

Priyadarsini, K. I. (2009). Photophysics, photochemistry and photobiology of curcumin: studies from organic solutions, bio-mimetics and living cells. J. Photochem. Photobiol. C Photochem. Rev. 10, 81-95. doi: 10.1016/j. jphotochemrev.2009.05.001

Qi, Y., Huang, Y., Li, B., Zeng, F., and Wu, S. (2018). Real-time monitoring of endogenous cysteine levels in Vivo by near-infrared turn-on fluorescent probe with large stokes shift. Anal. Chem. 90, 1014-1020. doi: 10.1021/acs.analchem. $7 \mathrm{~b} 04407$

Saroj, S., and Rajput, S. J. (2018). Composite smart mesoporous silica nanoparticles as promising therapeutic and diagnostic candidates: recent trends and applications. J. Drug Deliv. Sci. Technol. 44, 349-365. doi: 10.1016/j.jddst.2018. 01.014

Shi, J., Kantoff, P. W. J., Wooster, R., and Farokhzad, O. C. (2017). Cancer nanomedicine: progress, challenges and opportunities. Nat. Rev. Cancer. 17, 20-37. doi: 10.1038/nrc.2016.108

Tran, S., DeGiovanni, P. J., Piel, B., and Rai, P. (2017). Cancer nanomedicine: a review of recent success in drug delivery. Clin. Transl. Med. 6:44.

Warburg, O. (1925). The metabolism of carcinoma cells. J. Cancer Res. 9, 148-163. doi: $10.1158 /$ jcr.1925.148

Watermann, A., and Brieger, J. (2017). Mesoporous silica nanoparticles as drug delivery vehicles in cancer. Nanomaterials 7:189. doi: 10.3390/nano7070189

Wedege, K., Dražević, E., Konya, D., and Bentien, A. (2016). Organic redox species in aqueous flow batteries: redox potentials. Chem. Stab. Solubility. Sci. Rep. 6:39101.

Wicki, A., Witzigmann, D., Balasubramanian, V., and Huwyler, J. (2015). Nanomedicine in cancer therapy: challenges, opportunities, and clinical applications. J. Control. Release. 200, 138-157. doi: 10.1016/j.jconrel.2014. 12.030 
Yang, S., Zhao, L., Yu, C., Zhou, Z., Tang, J., Yuan, P., et al. (2006). On the origin of helical mesostructures. J. Am. Chem. Soc. Artic. 128, 10460-10466. doi: $10.1021 / \mathrm{ja} 0619049$

Youn, Y. S., and Bae, Y. H. (2018). Perspectives on the past, present, and future of cancer nanomedicine. Adv. Drug Deliv. Rev. 130, 3-11. doi: 10.1016/j.addr. 2018.05.008

Zanella, F., Lorens, J. B., and Link, W. (2010). High content screening: seeing is believing. Trends Biotechnol. 28, 237-245. doi: 10.1016/j.tibtech.2010.02.005

Zock, J. (2009). Applications of high content screening in life science research. Comb. Chem. High Throughput Screen. 12, 870-876. doi: 10.2174/ 138620709789383277
Conflict of Interest: The authors declare that the research was conducted in the absence of any commercial or financial relationships that could be construed as a potential conflict of interest.

Copyright $\odot 2021$ Freidus, Kumar, Marimuthu, Pradeep and Choonara. This is an open-access article distributed under the terms of the Creative Commons Attribution License (CC BY). The use, distribution or reproduction in other forums is permitted, provided the original author(s) and the copyright owner(s) are credited and that the original publication in this journal is cited, in accordance with accepted academic practice. No use, distribution or reproduction is permitted which does not comply with these terms. 